https://doi.org/10.46344/JBINO.2021.v10i2b.07

\title{
PROSTHODONTIC MANAGEMENT OF MID- FACIAL DEFECT - A CASE REPORT
}

Sruthi YSS', Rao BL2, Satyanarayana S V Tammineedi3 ${ }^{3}$ Pallavi $C^{4}$

1. Postgraduate student, Department of prosthodontics, Lenora institute of dental sciences

2. Professor \& Head, Department of prosthodontics, Lenora institute of dental sciences

3. Reader, Department of prosthodontics, Lenora institute of dental sciences

4. Senior Lecturer, Department of Prosthodontics, Sree Sai dental college \& research institute.

\begin{abstract}
Any maxillofacial deformity lowers the person's quality of life by affecting them in all grounds of life physically, psychologically, and socially. Rehabilitation of patients with these deformities is of great challenge to the Prosthodontist because it is the esthetics that has to be achieved, giving equal importance in these cases and the function. This case report illustrates maxillofacial prosthetic rehabilitation of significant midfacial defect, including orbit and its contents, zygoma, soft tissues, including half of the nose, cheeks of the right side orofacial communication, which resulted from resection of mucoepidermoid carcinoma. Various retentive aids were utilized, such as ear lobe of spectacles, magnets, adhesives, and acrylic to enhance the retention and provide the near to natural appearance and functioning.
\end{abstract}

KEYWORDS: Facial defect, Facial prosthesis, Silicone, Mucoepidermoid carcinoma, Prosthodontist, Magnets 


\section{INTRODUCTION:}

An ancient quotation, "love for life comes only after love for face," depicts the importance of face and beauty for a man. Any defect from a small dark spot or scar to a significant maxillofacial defect leads to a compromised quality of life by affecting them mentally, physically, and socially. These defects create a series of problems to restore because they affect esthetics, function, and psychology. The reason for these maxillofacial defects is either congenital or acquired due to resection of carcinoma, trauma, or gunshot injuries. The midfacial defects are classified by Marunick MT et al.,1 into 1.midline midfacialldefects, including the nose and upper lip, and 2.lateral defects, including the cheek and orbital contents. Maxillofacial reconstruction can be treated by surgical reconstruction by alloplastic or autogenous grafts or prosthesis rehabilitation, or a combination of both. Although the scope of plastic surgery has advanced so much, it is the Prosthodontist who restores the quality of life in little time and reduced hospitalization with a best predictable outcome, ${ }^{2}$ because prosthodontics "is the dental specialty about the diagnosis, treatment planning, rehabilitation, and maintenance of the oral function, comfort, appearance, and health of patients with clinical conditions associated with missing or deficient teeth and/or maxillofacial tissues by using biocompatible substitutes; comp, fixed prosthodontics, implant prosthodontics, maxillofacial prosthetics, removable prosthodontics" as per GPT 9.3 Factors to be considered for prosthodontic rehabilitation are 1. The amount of Remaining supportive tissue, 2. Age and medical condition of the patient, 3. Pathologic findings, 4. Psychological status of the patient to deal with a prosthesis, 5 . Number, position, and condition of the remaining teeth, and 6. Skills of the Prosthodontist. Through this paper, we would like to bring about the step-by-step procedure of rehabilitation of maxillofacial defects. 1

\section{CASE REPORT:}

A 55-year-old male patient reported to the Department of Prosthodontics, Lenora institute of dental sciences, Rajahmundry, India, for prosthetic rehabilitation of maxillofacial defects involving the face, orbit, and maxilla (Fig 1a). He had a history of resection of mucoepidermoid carcinoma from the hard palate, zygomatic arch, and orbital fossa on the right side with infratemporal fossa clearance (Fig 1b). Intra-oral examination revealed partially edentulous maxilla and palatal defect in continuation with the extra-oral defect. The patient complains of difficulty in social being an awkward feeling when people question him about the reason for deformity and treating him as a challenged person. He also complains that getting the surgical dressing being complicated and not economical (Fig la). All the treatment options were explained and discussed, keeping in mind the complaints of the patient and economic constraints silicone facial prosthesis was planned an entire treatment plan along with the limitations of the prosthesis was discussed with the patient, and consent discussed with the patient, and
w.jbino.com | Innovative Association 
was taken from the patient. As the patient underwent recent surgical and radiation therapy and due to insufficient healing of the wound, we planned only for the interim obturator to aid in closure of the palatal defect and aid in swallowing and phonetics, and continuation of surgical dressing of the extraoral fault until the complete healing of the surgical site. After eight months of the healing period, the surgical site was assessed. Treatment protocol (impression making, wax pattern, iris positioning, mold space preparation, prosthesis try-in, and insertion) was started. IMPRESSION PHASE:

Prior to making the impression, Vaseline was applied, and gauze was placed in the defect area to prevent the flow of the impression material into the undercut areas, and the defect was boxed with the wax sheets to confine and support the impression. Impression was made using alginate (irreversible hydrocolloid) (Fig 1c). The impression was boxed and poured using dental stone (Type III gypsum product Fig 1d).

WAX PATTERN AND IRIS POSITIONING:

The wax pattern (Fig le) was sculpted by observing the contralateral side, and try-in was done, and modifications were done clinically. Iris (stock eye of size equal to the contralateral side was selected) positioning was done using the contralateral eye as reference (Fig 1f, 2a). MOULD SPACE PREPARATION:

As the defect site was large and flasking in the regular flask is not possible, so the wax pattern was placed directly in the plaster mix. Notches were made on the four sides of the plaster mold (Fig 2b), and a separating medium was applied before the upper or second pour was done using dental stone (Fig 2c). After the second pour has set, dewaxing was done, leaving the mold space for the prosthesis. An adequate amount of silicone material was dispensed (R.T.V. silicone) on the glass slab. Manipulation of the silicone material was done in the presence of the patient for accurate shade matching. Intrinsic coloration did (Fig 2d). Silicone material was packed and kept for polymerization for 24 hours in a bench-press. After 24hours, molds were separated, the silicone prosthesis retrieved, and finishing done.

TRY-IN \& INSERTION:

The prosthesis try-in was done (Fig 2e), and as spectacles (Fig 2f) have opted as mechanical retentive aid, different spectacles were tried, and the one which masks the delineation between the prosthesis and the intact tissue surface was selected. Retention was planned using adhesive.

INTRAORAL DEFECT REHABILITATION:

Due to resection of the maxilla's right side intra-orally, there is the missing tooth in relation to $12,13,14,15,16,17$, and continuation to the extraoral defect. But after the placement of the facial prosthesis, the continuation was closed, and there is an improvement in the speech of the patient. So, the intraoral prosthesis was planned just like a conventional acrylic removable partial prosthesis without engaging in the defect. Clasps were only the aid of retention for the intraoral prosthesis

FOLLOW UP:

Follow-up appointments were planned for one week, one month, three months, six months. During the follow-up after one months. During the follow-up 
week, the patient's main complaint was prosthesis was stable during the static position or in the upright position, loss of retention, and frequent dislodgement of the prosthesis when bent forwards. The modifications planned were to use magnets and attach the acrylic block to the ear lobe of spectacles.

\section{ATTACHMENT OF MAGNETS AND ACRYLIC RETENTIVE BLOCK:}

Markings were made on the silicone prosthesis and the removable partial prosthesis individually in position while the counterpart is missing, and slots were prepared in the prosthesis. The magnets were placed (Fig 3a, 3b, 3c). The retention was enhanced after the placement of the magnets and the prosthesis retained in position even during functional movements and forward bending. The acrylic retentive block was attached to the legs of the spectacles (right side) to hold the prosthesis in intimate contact with the tissues in the region and aid in retention. For fabrication of acrylic retentive block, A wax block was prepared and tried for positioning and cured in clear acrylic resin, and shade matching was done in accordance with the prosthesis. (Fig $3 d, 3 e$ ) DISCUSSION:

In society, the patient with head and neck cancer may be confronted with three sets of crises. The first set is generated because he is ill. The second set of crises develops because the specific illness is cancer (tumor). The third group of crises are related to the deformity associated with the necessary therapeutic measuressurgery, radiation therapy, or chemotherapy. The cancer concerns cycle includes: guilt, depression, facial disfigurement, ostracism, anxiety, helplessness \& disability, chronicity, hopelessness, abandonment, inferiority. Surgery and radiation remain the primary modes of treatment for most tumors. Surgical resection of a tumor has the advantage of physically removing the bulk of malignant tissue, while radiation has the advantage of localizing morbidity to the specific area of the body. The choice between surgical reconstruction and prosthetic restoration of large facial defects is a difficult and complex decision, one which depends on the size and etiology of the defect and the wishes of the patient. A variety of circumstances may dictate prosthetic restoration of facial defects: (1) When a large resection is necessary and recurrence of tumor is likely, (2) Surgical restoration of large defects is technically difficult and requires multiple procedures and hospitalizations. Increasing numbers of the tumors in the region are being treated with radiation therapy. Reduced vascularity, increased fibrosis, and scarring of the tissues bordering the defect increase the risk of complications associated with reconstruction. (4) as a temporary prosthesis, it is fabricated during the surgical treatment interval. (5) carcinoma of the nasal structures is prone to reoccur, so prostheses are often suggested. 4,5

Alginate was selected as the making the impression instead of elastomers because alginate can flow to all the defect areas without any force application. As the defect site was large direct flasking was planned using the stone plaster. The prosthesis's intaglio surface was placed in the plaster's first pour before placing the the plaster's first pour before plac
w.jbino.com | Innovative Association 
surface is coated with a thin mix of the plaster. Once the plaster has reached the setting time, indentation notches were made on all four sides of the first pour plaster for reorientation. Once the material is completely set, a separating medium was applied, and boxing of the plaster block was done to hold the second pour of the mold; for the second pour, the dental stone was used to increase the strength of the mold and to record the fine details of the cameo surface of the prosthesis. Before the second pour, a thin mix of stone was applied to the wax pattern, and then the second pour was done.

Intrinsic coloration was opted to incorporate the lively look to the prosthesis. After try-in of the silicone prosthesis, it was cleaned with acetone. Extrinsic stains (Cosmesil) were added in single part silicone and applied over the prosthesis to match the contralateral side. A hairdryer was used to dry the extrinsic coloration. Though the defect is large and retention of the prosthesis is questionable, implants were not planned as retentive aids because of the patient's unwillingness of patients for another surgery and delay in the fabrication of the prosthesis, economic status, and tumor recurrence rate constraints of the patient. The other auxiliary aids used were spectacles and adhesives. But adhesives as retentive aids have a questionable role, so the prosthesis was provided with additional mechanical retention by using magnets in the silicone prosthesis and the removable partial prosthesis and addition of acrylic retentive block to the spectacles.

The silicone prosthesis's main limitation is the weight of the prosthesis and the difficulty in making it hollow and retaining the prosthesis. The problem of retention, in this case, has been overcome with the help of the mechanical aids of retention like magnets in the facial prosthesis and intaglio surface of the removable prosthesis with unlike poles of the magnets facing each other.4,5,6 Spectacles were modified by the addition of acrylic block to the legs on the defect side, holding the prosthesis intact to the tissue surface.

CONCLUSION:

Treatment options may vary according to the surgical site anatomy, size of the defect, recurrence rate of the tumor, undercuts present, patients' economic status (for selection of the material of the prosthesis, retentive aids), ease and knowledge of the dentist. The Prosthodontist's goal should strictly adhere to the MM De Vans dictum, i.e., perpetual preservation of what remains is of utmost importance than the exact replacement of what is lost. It is the Prosthodontist's duty to explain the advantages and the limitation of the materials and the treatment outcome, especially in meeting the patient's unrealistic expectation about the prothesis.

\section{ACKNOLEDGEMENT:}

I would like to acknowledge Dr. P.S.H.L. Parvathi (II yr. P.G. student), Dr. V. Chakradhar (I yr. P.G. student) for their constant support, and advices in all the aspects and Mr. Shaik Davood (CDT) for his timely support in the laboratory work. REFERENCES:

1. Marunick MT, Harrison R, Beumer J III: Prosthodontic rehabilitation of midfacial defects. J Prosthet Dent 1985; 54:553-60. 
2. Robinson JE. Prosthetic treatment after surgical removal of the maxilla and floor of the orbit. J Prosthet Dent 1963; 13:178-84.

3. The glossary of prosthodontic terms. J Prosthet Dent 2005; 94:10-92.

4. Sasaki $H$, Kinouchi $Y$, Tsutsui $H$, Yoshida $Y$, Karv $M$, Ushita T. Sectional prostheses connected by samarium-cobalt magnets. J Prosthet Dent 1984; 52:556-58.

5. Goiato MC, Fernandes $A U$, dos Santos DM, Barão VA. Positioning magnets on a multiple/sectional maxillofacial prosthesis. J Contemp Dent Pract 2007; 8:101-07.

6. Dumbrigue HB, Fyler A. Minimizing prosthesis movement in a midfacial defect: A clinical report. J Prosthet Dent 1997; 78:341-45.

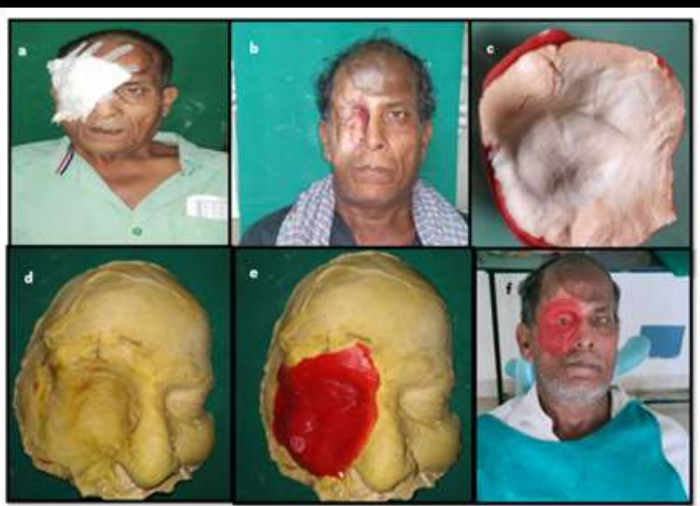

FIG 1: 1a. Pre-op photograph, 1b. Facial defect, 1c. Facial defect Impression, 1d. Cast, 1e. Wax pattern, 1f. Wax pattern try-in and iris positioning.

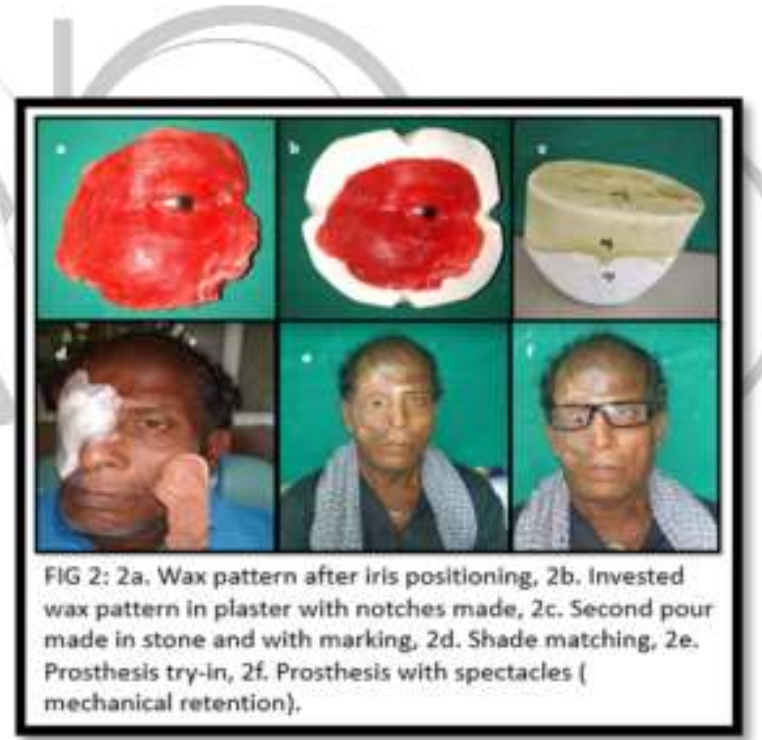

